\title{
Narrative Study of Emotion Marketing: The Uses Of Twitter At @Hijup (Www.Hijup.Com)
}

\author{
Putri Athia \\ (Economic and Business Faculty, Brawijaya University, Indonesia)
}

\begin{abstract}
Marketing once was only an afterthought to production. Marketing advises production about how much of the company's product to make and then tells logistics when to ship it. By the invention of internet and social media websites, marketing is completely changed. Marketers decided to put more attention and customer focused. This study is set forth to understand the role of Twitter as an inter alia media at @HijUp using Emotion Marketing concept. The indicator of Emotion Marketing is Emotional Es: Equity, Experience and Energy, and this paper focused on Equity only. This study is using qualitative research and analyzed through content analysis and divided into element of Emotion Es; Equity, Experience, and Energy. The main finding of this study is@ @ HijUp succeed to build and emotional connection with their customers via Twitter, as mentioned by inter alia media theory. This emotional connection comforts the customers and HijUp.com is able to bring the emotion into a strategic level. This study was only conduct by online questionnaires and online messenger, except Skype, due the tight schedule of informants and their gadget limitations. Some failed online questionnaire to targeted customers because of the short period of announcement. This paper offer insight the role of social media, especially Twitter and its connection with customers' emotion.
\end{abstract}

Keywords - Social Media, Twitter, Emotion Marketing, Internet, E-marketing

\section{INTRODUCTION}

Marketing once was only an afterthought to production. Marketing advises production about how much of the company's product to make and then tells logistics when to ship it. By the invention of internet and social media websites, marketing is completely changed. The invention the gadgets, tablets and smart phones, made every aspects of our lives are connected into internet. How we share, find a community and both connect and disconnect will give us unprecedented influence over our place in the world. Internet 2.0 Communities include chat rooms and list serves, instant messenger services and the online forums that let users post their opinions, ask questions, and contribute to online discussions.

One example of a powerful social media is Twitter. From www.wikipedia.com, Twitter is an online social networking service and microblogging service that enables its users to send and read text-based posts of up to 140 characters, known as "tweets". It was created in March 2006 by Jack Dorsey and launched that July. The service rapidly gained worldwide popularity, with over 300 million users as of 2011, generating over 300 million tweets and handling over 1.6 billion search queries per day. It has been described as "the SMS of the Internet." Twitter Inc. is based in San Francisco, with additional servers and offices in New York City. Twitter ranked 9 in Alexa Traffic, and 8 in US Rank.

Since its launch in 2006, Twitter has accumulated more than 175 million users in 2011, with recent growth described as 'explosive' (Lefkow, 2010). Twitter is, however, different from other marketing communications media, which can be classified inter alia, as one-to-one (e.g. e-mail), one-to-many (e.g. mass media) and many-to-many (e.g. the web and online groups) (Hoffman and Novak, 1996 at Burton and Soboleva, $2012)^{[1]}$. This uniqueness of Twitter makes it as apowerful social media for a organization, company, or a virtual community since it can reach many people in once.

Virtual Community define as online users which come together in cyberspace based on similar interests, transcending geographical and social boundaries. For example, one of communities that started from social medias is Hijabers Community. This community is consisting of women wearing hijab (veil) in their daily life and gather as one to spread the goodness of their faith.

Hijabers Community (HC) was first founded at Jakarta, and by the time, there're many of same community existed at other cities in Indonesia. The unique of this community is their great sense of fashion, proven the world that women with hijab are beautiful and up to date. This statement is encountered the paradigm that women with hijab are limited in style and less up date.

The internet impacts upon marketing in two main ways: distribution and communication. The first concerns distribution and marketing channels. The second element concerns the Internet as a communication medium (Fill, 2009:737) ${ }^{[2]}$. Because of HC great influence in fashion hijab and style, people started to seek their outfit at the market, and the demand is increasing. Catching the opportunities, the fashion hijab businesses are growing so fast. Supported by huge number of communities' member and their great influences which shared 
globally using Social Medias, HijUp.com is growing and gain quite good income. They sell many clothing and accessories from many well-known brands among Hijabers Community Members.

HijUp.com was selected because HijUp.com is the first moslemah online store which using one-stopshopping concept. HijUp.com is consists of several numbers of moslemah fashion designer which few of them are also the founder of Hijabers Community. HijUp.com already has a narrow target which is Hijabers Community and decided to use social media websites to stay connected with them.

One of social media website that HijUp.com use is Twitter with @ HijUp username, a micro blog where they are trying to build an image, getting more interactive with their customers, and keep the customers update with the latest stock, discount, or quizzes.

Since every community has a strong personal relationship among others and loyalty, this is indicates strong emotion that they already have. HijUp.com already supported by huge members of Hijabers Community, then how about elevates the emotion into a strategic level?

This idea of employ emotion to the marketing activity is called by Emotion Marketing. Emotion is intangible, irrational, mushy, but as force capable of inspiring customers to act, to buy, and to get attached with. Emotion Marketing is the enterprise-wide pursuit of a sustainable connection that makes customers feel so valued and cared for they will go out of their way to be loyal (Robinette et al. 2001:4) ${ }^{[3]}$. It elevates emotion to the strategic level, recognizing its importance in creating or strengthening a relevant brand identity and managing the consumers' experience. Emotion marketing includes making customers feel "cared for" and caring is essential for loyalty.

The model of Emotion Marketing is Value Star. These five points further break down the traditional value equation: Cost (Money and Energy) and Benefit (Product, Equity and Experience). The Value Star divides it into rational and emotional components. Money and Product are on the rational side because based on both customers are setting their priorities. In contrast, other three value drivers-Equity, Experience and EnergyHallmark called as Emotional Es. They not only provide opportunities for clear differentiation from the competition, but research shows they actually drive the majority of decisions to purchase.

Figure 1.1

Value Star

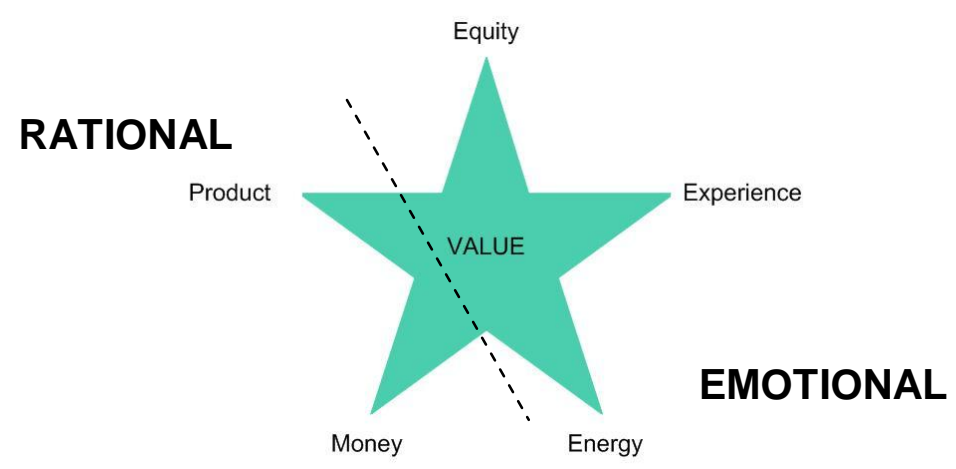

Resource: Robinette et al., Emotion Marketing (2001:21)

\subsection{Previous Related Research}

The previous research about Twitter study is from Burton and Soboleva $(2011)^{[4]}$ titled Interactive or reactive? Marketing with Twitter. Despite rapid growth in organizational use of Twitter, there is little theoretical or empirical research examining how different organizations use Twitter. This paper aims to analyze and compare use of Twitter in 12 accounts held by six organizations in the USA and Australia, drawing on existing models of interactive communications. The results demonstrate different ways in which the interactive capabilities of Twitter can be used to communicate with customers. However by also demonstrating lack of consistency in Twitter practice within most organizations, the results reinforce the need for strategic consistency in developing Twitter practice.

Previous study of Emotion Marketing is from Durkin et al. (2012) ${ }^{[5]}$ titled Emotional connections in higher education marketing. The paper aims to describe a brand re-positioning exercise and explore how an emotionally driven approach to branding can help create meaningful connections with potential undergraduate students and can positively influence choice. The finding is the use of an emotionally driven branding concept has positively impacted business development and brand likeability within a UK university.

\subsection{Research Question and Purpose}

As explained before, this paper presents the use of Twitter as the one of digital marketing tool on HijUp.com. The question of this study is How @ HijUp as an inter alia media build emotion to its followers by using Emotion Marketing Concept specifically at Emotional Es (Energy, Equity and Experience)? Then the 
purpose is to explore and describe the uses of Twitter for Emotion Marketing at @HijUp. At this stage of research, the Twitter will be generally defined as inter alia media.

Moreover, based on the phenomenon, the research gap in this study is how rare that similar research conducted and researched at this particular field. By the time this research wrote, researcher haven't found any quite similar research which held before, especially in Indonesia.

\section{LITERATURE REVIEW}

\subsection{Marketing}

Most people think of marketing as a way for a firm to make profits. In addition, marketing isn't useful only in big countries with well-developed economies. It can also jump-start the economies of less developed countries by actually creating markets, which is putting buyers and sellers together. Marketing often is designed to benefit an entire industry, which can help many firms simultaneously. Described at Grewall and Levy (2008), most successful firms today are market oriented. That means they generally have transcended a production or selling orientation and attempt to discover and satisfy their customers' needs and wants.

Before the turn of $21^{\text {st }}$ century, better marketing firms recognized that there was more to good marketing than simply discovering and providing what consumers wanted and needed; to compete successfully, they would have to give their customers greater value than their competitors did. Value reflects the relationship of benefits to costs, or what you get for what you give and firms become value driven by focusing on three activities: Sharing information, Balancing benefits with costs, and Building relationship with customers (Grewall and Levy, 2008) ${ }^{[6]}$.

\subsection{Social Media}

As the dramatic changes and impact that technology caused nowadays, digitisation provides marketers to develop new ways to communicate with customers. The key forms of digital media are: the internet, database technologies, multimedia, mobile technologies, business applications, interactive television, video conferencing and electronic kiosks.

Digital media allows customers for true interactivity based on communications so that organisations can build emotions through it. Digital media also enable organisations to do multichannel marketing with cheaper budget. Personalisation, mobility, and speed are providing by these digital medias as well. So that organisations can improve their efficiency and enhanced the relationships to the customers. The differences between traditional media and digital media are shown below:

Table 2.1

Comparison of new and traditional media

\begin{tabular}{|c|c|}
\hline Traditional Media & New Media \\
\hline One-to-many & One-to-one and many-to-many \\
\hline Greater monologue & Greater dialogue \\
\hline Active provision & Passive provision \\
\hline Mass marketing & Individualized marketing \\
\hline General need & Personalised \\
\hline Branding & Information \\
\hline Segmentation & Communities \\
\hline \\
Resource: Fill, Marketing Communications: Interactivity, communities and content (2009: 759)
\end{tabular}

The Internet is a network of computer networks. Internet is a good example of hypermedia. Like new media before it, Internet will forever change people who use it. Price and Price (2002) on Borchers $(2005: 11)^{[7]}$ explained that hypermedia fundamentally change the relationship between persuader and audience member. With the invention of Internet, there is no longer one persuader who provides information to a mass audience. In Indonesia there are 55 million internet users, 8 world rank according to Internet World Stats and managed in 20 top countries aside with China and States.

One main characteristics of Internet is interactivity. The first phase of Internet is web 1.0 which was less interactive than web 2.0. The Web 1.0 was complex, text based, and need an expert to run it. Here in web 1.0 , internet users are treated as an audience and receive the information only. Web 1.0 was developed into web 2.0 that more interactive and user friendly.

Web 2.0 is place where users can take place in a two-ways communication and get into interactivity. Web 2.0 gave us the social media sites such as Facebook, blog, Twitter and Youtube. Around 2005, based on Fuchs (2008), web 2.0 fully emerged fully emerged and that the web has entered a new phase of development.

Social media websites defined on Sterne $(2010)^{[8]}$ as a medium which allows anybody to communicate with everybody on the Internet. Social media websites have a great influence on people lives because these three 
assumptions of media: media persuade people, media present people with mediated realities, and media are profit-making businesses (Borchers, 2005:101). A particular social media described at this paper is Twitter.

Twitter is an online social networking service and microblogging service that enables its users to send and read text-based posts of up to 140 characters, known as "tweets". It was created in March 2006 by Jack Dorsey and launched that July. The service rapidly gained worldwide popularity, with over 300 million users as of 2011, generating over 300 million tweets and handling over 1.6 billion search queries per day. It has been described as "the SMS of the Internet." Twitter Inc. is based in San Francisco, with additional servers and offices in New York City. Twitter ranked 9 in Alexa Traffic, and 8 in US Rank.

Twitter, basically, is a powerful mobile social network which enables the users to keep up with people, businesses, and organizations they are interested in. No personally known is needed. In turn, other users can elect to follow your updates. Twitter is an internet medium which allows the users to do both of one to one communication and mass communication. This is why Twitter unique and different to any other social media websites. Twitter changed and enhanced the way people communicate with each other, with brand and companies, and with social movements and initiatives. Twitter has ability to empowered users raising money for people in need; coordinate rescue efforts in the wake of a natural disaster, traffic reports, live tweet from concert or events, and many more. In sum, Twitter is a service for friends, family, and coworkers, to communicate and stay connected through the exchange of quick, frequent messages and users write short updates, often called 'tweets' of 140 characters or fewer.

The functions of Twitter for business or other profit organization are first Twitter can facilitate excellent customer relations. By posting the products information, feedback to any complaints and testimonials, solve problems, lead to increase the sales. Second a presence in Twitter helps avoid bad customers. In many cases, when twitter user post while they are disappointed upon a service or product, the representative could replied it quickly. This is make customers feel like they are have a voice, and being heard.

Twitter as an inter alia media means Twitter can be classified as an inter alia, which is one-to-one (e.g. e-mail), one-to-many (e.g. mass media) and many-to-many (e.g. the web and online groups) (Hoffman and Novak, 1996 at Burton and Soboleva, 2012). This uniqueness of Twitter makes it as apowerful social media for a organization, company, or a virtual community.

\subsection{HijUp.com}

At the present, the fashion hijab businesses specifically in Indonesia are growing so fast.HijUp.com is the first moslemah online store which using one-stop-shopping concept. HijUp.com is consists of several numbers of moslemah fashion designer which few of them are also the founder of Hijabers Community. They sell branded and high quality product from moslemah desginers. HijUp.com already has a narrow target which is the members of Hijabers Community and integrated social media websites to stay connected with them. Their Twitter username is @ HijUp, a micro blog where they are trying to build an image, getting more interactive with their customers, and keep the customers update with the latest stock, discount, or quizzes.

\subsection{Emotion Marketing}

Emotion is difficult to defined, because it's not easy for people to say what they do feel inside. Emotion basically is a feeling or affect, which occurs when a person is in a state or an interaction that is important to an individual, especially to his or her well being (Campos et al. at Santrock, 2008:155) ${ }^{[9]}$. Some psychologists argue that emotions are the "glue" that connects people's life events.

Emotion Marketing is the enterprise-wide pursuit of a sustainable connection that makes customers feel so valued and cared for they will go out of their way to be loyal (Robinette et al. 2001:4). It elevates emotion to the strategic level, recognizing its importance in creating or strengthening a relevant brand identity and managing the consumers' experience. Customer loyalty is vital to success for one important reason: it's directly related to profit.

Emotion marketing includes making customers feel "cared for" and caring is essential for loyalty. The more company shows which they are really care, the more loyal customers appear to be. Caring is the bridge between satisfaction and loyalty. Emotion marketing is proven and practical way to let the customers know that the company is genuinely caring.

The Emotion Marketing Model is Value Star. 


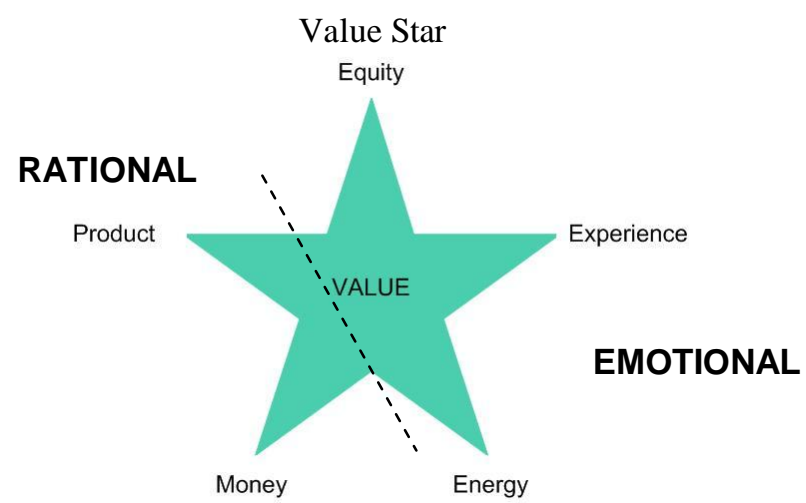

Resource: Robinette et al., Emotion Marketing (2001:21)

These five points further break down the traditional value equation: Cost (Money and Energy) and Benefit (Product, Equity and Experience). The Value Star divides it into rational and emotional components. Money and Product are on the rational side because based on both customers are setting their priorities. In contrast, other three value drivers-Equity, Experience and Energy-Hallmark called as Emotional Es. They not only provide opportunities for clear differentiation from the competition, but research shows they actually drive the majority of decisions to purchase. In this study we focused on Emotional Es, as described below.

a. Emotional Es: Equity

Equity is a combination of the trust a brand earns and an identity that allows consumers feel emotionally connected to it. In business, equity is all about trust. Equity is where identity and image meet. Identity is what company aspires to be, while image is how consumers actually perceive the brand. The Brand Identity consist of company's aspirations, personality, and consumer promises. Companies that have successfully established trust with their customers and have built their Equity emotion have the permission to extend into new product lines, expand into new industries, and command price premiums

b. Emotional Es: Experience

Experience based on Robinette et al., (2001:59) is the collection of points at which companies and consumers exchange sensory stimuli, information, and emotion. These exchanges divide into three categories: Transactional exchanges, when the product or service delivered and payment made; Informational exchange, when rational data is shared; Emotional exchanges or touch points, when the company and consumer connect emotionally

c. Emotional Es: Energy

Time, is the one and only resources which cannot be replaced. Once it has gone, it has gone forever and not coming back. People are concern about using time more efficient and they are not welcome for waiting. This concern is sending a message to the customers that company is valued the time as much as the customers do. With efficiently consuming time, company also decrease the customers' energy consuming. Some ways to conserve time and energy according Robinette et al. are: make things more accessible and easier; make things worthwhile; make things personalized; leverage information and technology; provide security.

\subsection{Prepositions}

The purpose of this case study was to understand the role of Twitter as an inter alia media using Emotion Marketing concept specifically at Emotional Es (Equity, Experience and Energy). Thus, prepositions for this study are described below.

a. Twitter as an inter alia media can be used to build Equity

b. Twitter as an inter alia media can be used to build Experience

c. Twitter as an inter alia media can be used to build Energy

\subsection{Research Design}

\section{RESEARCH METHOD}

This study is using qualitative research, which according to Lincoln and Gubba (1985) on Creswell $(1994: 4)^{[10]}$ is termed the constructivist approach or naturalistic. Qualitative research is selected because for researcher, the only reality is that constructed by the individuals involved in the research situation. Here, researcher interacts with that being researched. By going directly to the social phenomenon under study and observing it as completely as possible, researcher can develop a deeper and fuller understanding of it. 


\subsection{Case Study}

Four designs frequently found in human and social science research based on Creswell (1994:11) are Ethnographies, Grounded Theory, Case Studies and Phenomenological Studies. Because this study is started from a Twitter as an Internet phenomenon specifically on HijUp.com Twitter Account (@HijUp), this study is using Case Study. Case study can be classified as explanatory, exploratory, or descriptive. Explanatory case studies are best suited for answering the "why" and "how" questions. This study is categorized as explanatory research because the purpose of this study is how Emotional Marketing employed in @ HijUp Twitter Account.

Explanatory case study is useful when conducting causal studies (Berg 2001: 230) ${ }^{[11]}$. Explanatory case study is particularly use in complex studies of organizations or communities; one might desire to employ multivariate cases to examine a plurality of influence. These explanations in our case are referring to @ HijUp and the role of Emotional Marketing.

\subsection{Unit of Analysis}

The unit analysis of this research is @ HijUp. Twitter is one of the social media websites using by HijUp.com, and the username is @ HijUp. HijUp.com is already have a narrow target—Hijabers Communitybut still important for them to stay connected and build a strong emotional relationship with them. This makes the followers of @ HijUp as a potential buyer for HijUp.com.

\subsection{Validation requirements}

Yin (2009:40) ${ }^{[12]}$ proposed four tests which have been commonly used to establish the quality of any empirical research. Yin also provides each test with several tactics when doing the case studies. The tests are explained at the table below:

Table 3.1

Case Study Tactics for Four Design Tests

\begin{tabular}{|c|c|c|}
\hline Tests & Case Study Tactic & $\begin{array}{l}\text { Phase of research in } \\
\text { which tactic occurs }\end{array}$ \\
\hline Construct Validity & $\begin{array}{ll}\text { - } & \text { Use multiple sources of evidence } \\
\text { - } & \text { Establish chain of evidence } \\
\text { - } & \text { Have key informants review draft } \\
\text { case study report }\end{array}$ & $\begin{array}{l}\text { Data Collection } \\
\text { Data Collection } \\
\text { Composition }\end{array}$ \\
\hline Internal Validity & $\begin{array}{ll}\text { - } & \text { Do pattern matching } \\
\text { - } & \text { Do explanation building } \\
\text { - } & \text { Address rival explanations } \\
\text { - } & \text { Use logic models }\end{array}$ & $\begin{array}{l}\text { Data analysis } \\
\text { Data analysis } \\
\text { Data analysis } \\
\text { Data analysis }\end{array}$ \\
\hline External Validity & $\begin{array}{ll}\text { - } & \text { Use theory in single-case studies } \\
\text { - } & \text { Use replication logic in multiple-case } \\
\text { studies }\end{array}$ & $\begin{array}{l}\text { Research design } \\
\text { Research design }\end{array}$ \\
\hline Reliability & $\begin{array}{ll}- & \text { Use case study protocol } \\
\text { - } & \text { Develop case study database }\end{array}$ & $\begin{array}{l}\text { Data collection } \\
\text { Data collection }\end{array}$ \\
\hline
\end{tabular}

Resources: Yin, Case study research: design and methods, fourth edition (2009:40)

Construct validity is identifying correct operational measures for the concepts being studied. The goal is to ensure that the researcher's understanding of the constructs genuinely reflects the views of the respondents. Suggested tactics for increasing construct validity are: using multiple sources of evidence, establishing a chain of evidence and having key informants review the draft case study report. Which in this research, researcher adopt the three tactics of construct validity.

Internal validity, due to Yin (2009:40) is applied only for explanatory studies only; it's seeking to establish a causal relationship, whereby certain conditions are believed to lead to other conditions, as distinguished from spurious relationships. Internal validity is achieved when the causal relationships between variables are accurately identified.

Internal validity is mainly a concern for explanatory case studies, when a researcher is trying to explain how and why event $x$ led to event $y$. Is the researcher incorrectly concludes that there is a causal relationship between $x$ and $y$ without knowing that some third factor-z--may actually have caused $y$, the research design has failed to deal with some threat to internal validity.

External validity test is dealing with the problem of knowing whether a study's findings are generalizable beyond the immediate case study. Critics typically state that single case offer a poor basis for generalizing. However, such critics are implicitly contrasting the situation to survey research, in which a sample is intended to generalize to a larger universe. 
This analogy to samples and universes is incorrect when dealing with case studies. Survey research relies on statistical generalization, whereas case studies rely on analytic generalization. In analytical generalization, the researcher is striving to generalize a particular set of results to some broader theory.

Reliability is demonstrating that the operations of a study — such as data collection procedures - can be repeated, with the same results. There are two specific tactics to deal with reliability which using in this research: case study protocol and a development of a case study database. The general way of approaching the reliability problem is to make as many steps as operational as possible and to conduct research as if someone were always looking over the shoulder.

\subsection{Methodology}

In Case Study, the researcher needs to select a site or sites to study. The chosen site for this case study is @ HijUp and their followers. The exploration also continuing in HijUp.com and this study is conduct on May 2012. Access is provided by a gatekeeper who is the initial contact for the researcher and leads the researcher to other participants (Hammersley and Atkinson 1995) on Creswell (2007:125). In this study, the gate keeper is referring to the head of Marketing Department of HijUp.com.

\subsubsection{Informants characteristics}

The criteria of the participants which are interviewing for this study are:

1. Female

2. Moslem and using Hijab

3. The age range is between $18-30$ years old

4. Member of Hijabers Community or another similar hijab community at informant's living place

5. Non-member of Hijabers Community but aware about Hijabers and follow their trends

6. Twitter user and follow HijUp's Twitter account (@HijUp)

7. Been shopping on HijUp.com between April-May 2012.

As emphasized at Creswell (1994), unlike the quantitative research, qualitative research has a saturation point which indicates the researcher to stop collecting data. Saturation point achieved when the research question is answered, not when researcher think the data was saturated enough. The number of informants are depends on the saturation point, and in this study the saturation point was achieved at informants the eighth.

\subsubsection{Measurements}

Measurement here is according to the definition of Equity, one of Emotional E's element which are using to dig the Emotional Marketing deeper in this study. Equity is a combination of the trust a brand earns and an identity that allows consumers to feel emotionally connected to it. In business, equity is all about trust. Equity is where identity and image meet. Identity is what company aspires to be, while image is how consumers actually perceive the brand. The Brand Identity consist of company's aspirations, personality, and consumer promises. This should be timeless and consistently communicated across all media and consumer interactions.

\subsubsection{Research Instruments}

Prior to data collection, three research instruments were prepared: case study procedures, case study protocol, an interview guide, and a structure for a research database. The explanation is below:

- Researcher it self

- Case Study Procedures

- Case Study Protocol

- Online Questionnaires

- Structured research database

\subsection{Data Analysis}

In this study, the data analysis processing conducted as an activity simultaneously with data collection, data interpretation, and narrative reporting writing. In this particular activity, qualitative analysis clearly differs from quantitative approach. In this research, researcher will be using initial coding or open coding to begin the qualitative field analysis. The coding will be doing by computer data basing which is easier for researcher.

The conjoint activities which doing while researcher coding is memoing. The Coding and Memoing also conduct for the result of observation and documentation. The observation of @ HijUp timeline consists of testimonials, questions, statement of delivered package, complaints, HijUp surveys, and quizzes. It will be coding and memoing as well as the result of the online questionnaire. 
For Documentation, researcher will be coding and memoing the documents and e-mail correspondence between researcher and HijUp.com. Because HijUp.com also release Hijab Video Tutorials on the Youtube, researcher will be also coding and memoing the audio visual materials.

After coding and memoing, researcher will analyze the data by using Content Analysis. Content Analysis refers to Babbie $(2005: 328)^{[13]}$ is the study of recorded human communications. Among the forms suitable for study are books, magazines, web pages, newspapers, e-mail messages, as well as any components or collection thereof. Content analysis is essentially a coding operation.

Based on Singleton and Straits (2005:371) ${ }^{[14]}$, Content Analysis is a really set of methods for analyzing the symbolic content of any communication. The basic idea is to reduce the total content of a communication (e.g. all of the words or all the visual imagery) to asset of categories that represent some characteristic of research interest. Thus, content analysis may involve the systematic description of either verbal or nonverbal materials.

Then, researcher will represent the report by using a diagram, or visual representations of relationships between concepts. These data displays that present information visually and systematically in the form of various types of matrices, graphs, charts, and networks.

\section{FINDINGS}

The purpose of this case study was to understand the role of Twitter as an inter alia media at @ HijUp using Emotion Marketing concept specifically at Emotional Es (Energy, Equity, and Experience). The study examined the personal experience of @ HijUp followers who shopped at HijUp.com during April-May 2012.

In order to fully understand the reality, the following research question was used to identify Emotional Es. The research question is how @ HijUp as an inter alia media build emotion to its followers by using Emotion Marketing Concept specifically at Emotional Es (Energy, Equity and Experience). This research question was then broken down into twenty one questions which were posed to the informants.

The keynote consisted of definition of Emotion Marketing Concept, inter alia media, @HijUp, and Emotional Es (Equity, Experience and Energy). This Chapter consists of informants profile and the explanation about Emotion Marketing specifically at Emotional Es in order to answer the research question. The compositions of total 8 informants are: two informants from HijUp.com, one is the Managing Director and the other one is the Administrator of @ HijUp, six of the rest are the customers of HijUp.com.

Equity is where brand identity and brand image meet. Brand identity is what company aspires to be. Brand image is how consumers actually perceived the brand. Companies that have successfully established trust with their customers and have built their Equity emotion have the permission to extend into new product lines, expand into new industries, and command price premiums.

Brand Identity which aspired by HijUp.com is shown below.

Table 4.1

Online Questionnaire Quote (Equity)

\begin{tabular}{clll}
\hline Informants & \multicolumn{1}{c}{ Question } & \multicolumn{1}{c}{ Answer } & \multicolumn{1}{c}{ Keywords } \\
\hline $\begin{array}{c}\text { Managing } \\
\text { Director }\end{array}$ & $\begin{array}{l}\text { If HijUp is a human being, } \\
\text { what personality it might } \\
\text { be? }\end{array}$ & $\begin{array}{l}\text { Inspiring, smart, fun, stylish, } \\
\text { cheerful }\end{array}$ & $\begin{array}{l}\text { HijUp.com, inspiring, smart, } \\
\text { fun, stylish, cheerful }\end{array}$ \\
\hline
\end{tabular}

HijUp is stands from Hijab Up which wants to inspired moslemah (hijab) and result a higher level of confidence (Up). HijUp.com provides a way for moslemah to keep stylish even they have to cover their body. From the keywords on the table above, can be concluded that the identity which aspired by HijUp is a moslemah online store that inspired its' customers in becoming smart, fun, stylish and cheerful. Managing Director and HijUp's team visualized and managed this identity through their website, Twitter accounts, and other.

Table 4.2

Online Questionnaire Quote (Equity)

\begin{tabular}{ccll}
\hline Informants & \multicolumn{1}{c}{ Question } & \multicolumn{1}{c}{ Answer } & \multicolumn{1}{c}{ Keywords } \\
\hline \multirow{2}{*}{$\begin{array}{c}\text { What kind of image } \\
\text { Administrator }\end{array}$} & $\begin{array}{c}\text { HijUp.com wants to build } \\
\text { via @ } @ \text { HijUp? }\end{array}$ & $\begin{array}{l}\text { HijUp.com main value, which } \\
\text { is THE (Trusted, Helpful, } \\
\text { Empower) }\end{array}$ & $\begin{array}{l}\text { Image @ HijUp, THE } \\
\text { (Trusted, Helpful, Empower) }\end{array}$ \\
& & Ematched with our & \\
\hline
\end{tabular}

From the table above, we can understand that the image which @ HijUp caring and aspired to be is THE; stands from Trusted, Helpful and Empower. Twitter is the extension of HijUp.com and the information flow at Twitter is faster than at other media, the Administrator forced to be responsive to any tweet from 
customers. By being responsive to any tweet from customers, the Administrator is implementing the main value of HijUp.com which is THE (Trusted, Helpful and Empower).

Table 4.3

Online Questionnaire Quote (Equity)

\begin{tabular}{|c|c|c|c|}
\hline Informants & Question & Answer & Keywords \\
\hline Informant \#1 & $\begin{array}{l}\text { If HijUp is a human being, } \\
\text { what personality it might } \\
\text { be? }\end{array}$ & Humble, responsive, creative & $\begin{array}{l}\text { Personality, humble, } \\
\text { responsive, creative }\end{array}$ \\
\hline Informant \#2 & $\begin{array}{l}\text { If HijUp is a human being, } \\
\text { what personality it might } \\
\text { be? }\end{array}$ & Bubbly, smart, friendly & $\begin{array}{l}\text { Personality, bubbly, smart, } \\
\text { friendly }\end{array}$ \\
\hline Informant \#3 & $\begin{array}{l}\text { If HijUp is a human being, } \\
\text { what personality it might } \\
\text { be? }\end{array}$ & Feminine, modern & $\begin{array}{l}\text { Personality, feminine, } \\
\text { modern }\end{array}$ \\
\hline Informant \#4 & $\begin{array}{l}\text { If HijUp is a human being, } \\
\text { what personality it might } \\
\text { be? }\end{array}$ & Fresh, smart, modern & $\begin{array}{l}\text { Personality, fresh, smart, } \\
\text { modern }\end{array}$ \\
\hline Informant \#5 & $\begin{array}{l}\text { If HijUp is a human being, } \\
\text { what personality it might } \\
\text { be? }\end{array}$ & $\begin{array}{l}\text { Good moslemah who still } \\
\text { covered their body but not put } \\
\text { fashion behind }\end{array}$ & $\begin{array}{l}\text { Personality ,good moslemah, } \\
\text { fashion }\end{array}$ \\
\hline Informant \#6 & $\begin{array}{l}\text { If HijUp is a human being, } \\
\text { what personality it might } \\
\text { be? }\end{array}$ & Perfecto & Personality, perfecto \\
\hline
\end{tabular}

From six quotes above, there are several keywords that mentioned more than once by the informants. Those keywords are bubbly/friendly, smart and modern. These keywords proof that the image which captured by the followers and customers is quite same.

If we linked the image with the aspired identity of @HijUp, the THE values (Trusted, Helpful and Empower), the trusted value can be found from keyword responsive, helpful value can be identify from keyword responsive and friendly, and Empower value can be identify from keyword good moslemah, smart, and modern.

In sum, the brand identity which aspired by HijUp.com and @HijUp is match with the brand image captured by their customers and followers. This statement is supported by the visitor number of HijUp.com which reaches 7,000 people a day as quoted below.

“... 7000 visitors/day for daily traffic at HijUp.com.” (Managing Director)

The preposition for this element is Twitter as an inter alia media can be used to build Equity. The proposition for this element is as an inter alia media can be used to build Equity. Moreover, the findings for this element are Twitter can be a tool to control and maintain the image which has been built.

The second element of Emotional Es is Experience. Experience is the collection of points at which companies and consumers exchange sensory of stimuli, information, and emotion (Robinette et al, 2001:60). These exchanges fall into three categories:

(1) Informational exchange, when rational data is shared or when the product or service information is delivering from the company or sales person to the customers with the better communications.

Despite all of the strengths like faster and globally reach, e-commerce also has weaknesses, such as buyer cant touch the clothing material if it is clothing shopping or even fitting the clothes. As The Managing Director emphasized on her online questionnaire about reducing insecurity of HijUp.com customers by providing complete and easy to understand product information. 
Table 4.4

Online Questionnaire Quote (Experience: Informational Exchange)

\begin{tabular}{llll}
\hline Informant & \multicolumn{1}{c}{ Question } & \multicolumn{1}{c}{ Answer } & \multicolumn{1}{c}{ Keywords } \\
\hline & $\begin{array}{l}\text { How HijUp.com reduce the } \\
\text { customers' insecurity } \\
\text { related to online shopping? }\end{array}$ & $\begin{array}{l}\text { By moving experience offline } \\
\text { to online. If in offline shopping } \\
\text { customers can easily see the } \\
\text { clothes detail, then we provide } \\
\text { detailed product pictures with } \\
\text { high resolution. }\end{array}$ & $\begin{array}{l}\text { Moving } \text { experience offline to } \\
\text { online, detailed product } \\
\text { pictures, resolution }\end{array}$ \\
$\begin{array}{l}\text { Managing } \\
\text { Director }\end{array}$ & $\begin{array}{l}\text { What HijUp.com do to } \\
\text { make measurement and size } \\
\text { easy for the customers? }\end{array}$ & $\begin{array}{l}\text { Provide a clear measurements } \\
\text { and size information. }\end{array}$ & $\begin{array}{l}\text { Measurement, size, clear } \\
\text { information }\end{array}$ \\
& $\begin{array}{l}\text { What HijUp.com usually } \\
\text { does before uploading the } \\
\text { product pictures? }\end{array}$ & $\begin{array}{l}\text { Editing, resizing and add } \\
\text { watermark }\end{array}$ & Photo editing \\
\hline
\end{tabular}

From the table above can be concluded that to fulfill the informational exchanges, HijUp.com took some steps. First, to reduce the insecurity level of customers that might seldom doing online shopping, HijUp.com trying to bring the exact same experience from offline to online. By providing detail photos product with good resolutions and complete measurements info, HijUp.com hopes that it could bring the same experience as customers get on offline shopping.

Informational Exchange also handled by @HijUp. According to the Administrator, the information they have to tweet and spread are Product Information (stock, new arrival, and discount), Shopping Guide, Recent News about HijUp.com (event or new tenants), Quotes (words or from Holy Quran), Beauty and Tweet Interview with hashtag \#HijUpTalk.

From the Online Questionnaires, concluded that the information about products including \#ClearanceSale, \#HijUpNewArrivals and \#HijUpStock is in the first place, chosen by 4 informants. The rest of informants choose \#HijUpTalk and \#HijUpQuiz for the most beneficial information. 6 informants have exact same answers, which is @ HijUp is giving very update information related to HijUp.com and product stock. Two previews from both HijUp.com sides and @HijUp sides proven that HijUp.com and @ HijUp are success to deliver and inform the beneficial information for their customers and followers.

(2) Transactional exchanges, when the product or service delivered and payment made.

Transactional exchange is concerned with functional interactions between the customer and the product, with the main aim being to achieve high-sales volumes through effective management of the marketing mix.

The bond between customers and company is built when the first transaction is made. By providing good transactional exchanges, the bond is started nicely and customers are expecting to repeat the sales. Reading and ordering from a catalog is when transactional exchange occurs.

HijUp.com providing the customers with a clean and simple transaction flow. The entire of transactions are written in Bahasa Indonesia and English also easy to understand. HijUp.com ordered @ HijUp to continuously tweeting about \#HijUpGuide consists of online shopping guidance in HijUp.com. Hijup.com provides the consumers with HijUp Shopping Guide Video which can be accessed at http://www.youtube.com/watch?v=Wjz9xczN6RE.

(3) Emotional exchanges or touch points, when the company and consumer connect emotionally.

Receiving an e-mail from a service representative making sure that the customer's pleased with the purchase or getting a thank-you note from the company is an emotional exchange or touch point. Touch point also delivered to the customers by simply put some funny emoticon at their tweet, as shown below.

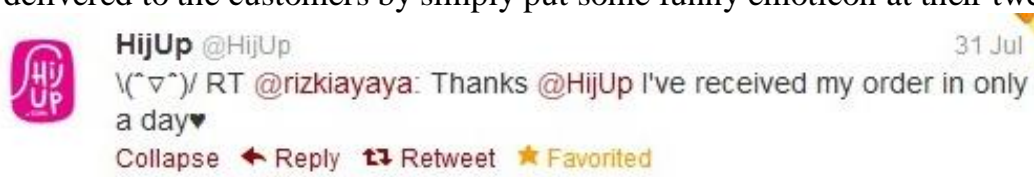

The preposition for this element is as an inter alia media can be used to build Experience. The proposition for this element is as an inter alia media can be used to build Experience. Twitter is not only can be used to build Experience, but also succeed to bring and deliver the information, transaction, and emotion to its customers. 
Energy may not appear to have the strong emotional ties that Equity and Experience have — but it does. Companies that demonstrate concern about people's time strengthen the connection they share. One of the Ecommerce strengths is convenient; buyers don't have to face the traffic, parking, or walk through stores which are consumed much energy. This is the basic why energy is quite important to build emotion connection with customers. According to Robinette et al. (2001), some points to consider under Energy are:

(1) Make things more accessible

It is not only using multiple channels to offer their products and services, but also giving consumers ability to make purchases wherever, whenever and however they want. HijUp.com is a business based online which can be accessed anywhere, from private PC at home to a mobile tablet or smart phone

(2) Make things easier

Businesses that can take over steps from customer-or take steps out altogether-save people's time by giving them less to do. Of course, internet sites have been the leaders in making things faster and easier. But it also easier for consumers abandons their site if the interface is too confusing or if there are too many steps to order.

HijUp.com solved this problem by designed a clean and simple interface. HijUp.com using a white based web and pink ornaments with sharp fonts which are designed to make consumers easier pick their selections. HijUp.com also ordered @ HijUp to continuously tweeting about \#HijUpGuide consists of online shopping guidance at HijUp.com

(3) Make things worthwhile

An extraordinarily strong product or service gives consumers the confidence that once they make a purchase, they won't waste any more Energy on it. Several tenants which selling their products at HijUp.com are Jenahara, Casa Elana, and Ria Miranda. These three designers are famous among members of Hijabers Community. These designers might have a premiums price, but the Equity of HijUp.com is already high, then the customers are willing to pay the price. Supported by the strong experience element, customers are trusting HijUp.com about the quality and service

(4) Make things personalized

A customized solution process is impressive to consumers. Personalization also has the benefit of making consumers feel valued; after all - the company is speaking directly to them, perhaps even using their names - and providing what's uniquely important to their lives.

Twitter, as an inter alia media, can be used as one-to-one communication tool with consumers. This makes them feel being heard and personalized, because it links directly to their Twitter account which accessed from their palm (smart phone/tablet). This, literally, company is speaking directly to them

(5) Leverage Information and Technology

One of HijUp.com value is empower the consumers. They empower the consumers by get them involved in every stages of transaction they made. Twitter, also can be used as an empowerment for HijUp.com

(6) Provide security

Many internet users harbor doubts about the privacy of their personal and credit information, leading them to drop out of the process before they make a purchase. A company in this position must work hard to connect with people, earn their trust, and clearly define privacy policies to calm any anger. Security, in this case, becomes a critical factor in building Energy.

In this case, HijUp.com provides it consumers by a clear process of transaction. HijUp.com continuously repeats the order and guidance to strengthen the security. HijUp.com sends e-mail confirmation to the shoppers to ensure them with a high security level in store. After shoppers transferred the money both via bank transfer or credit card, shoppers send a confirmation letter back to HijUp.com. Then HijUp.com sends the package to shoppers' address.

HijUp.com also already verified by PayPal, a well-known payment method online which has strict criteria's of online store. Online store verified by PayPal is one of a guaranteed to shop online.

The preposition for Energy element is Twitter as an inter alia media can be used to build Energy. The proposition for Energy element is Twitter as an inter alia media can be used to build Energy. Other findings for this element are by effectively managing people's Energy implies that all the exchanges with the company are interesting and engaging to the customer.

\section{CONCLUSION}

This study set forth to understand the role of Twitter as an inter alia media on @ HijUp using Emotion Marketing concept specifically at Emotional Es (Equity, Experience and Energy). A several numbers of online questionnaires were held with Managing Director of HijUp.com, @HijUp administrator, and @ HijUp followers and customers.

Since Equity is where the brand identity and image meet, HijUp.com via @ HijUp already matched the 
brand identity and brand image perceived by customers. The statements from HijUp Managing Director and @ HijUp administrator quoted before compared with the statements from @ HijUp followers are quite matches. HijUp.com and @ HijUp aspired to be Trusted, Helpful and Empower, thus the followers found HijUp.com and @ HijUp are helpful enough, responsive and informative.

Complete information about product description and specification, clean and simple transaction flow, completed by a touch point via twitter, HijUp.com is success to implement the Experience element. It has proven by 7,000 visitors a day at HijUp.com and almost 100 million worth transaction a day.

These sensory, rational, and emotional exchanges layer on top of one another and come together with remarkably moving results. Companies should orchestrate each component of Experience, so they all align with each other. As shown by HijUp.com by providing complete information from display, catalog, transaction and touch point emotion. If experiences can meet people's basic human needs, then consumers will reward the company with loyalty, as shown at @ HijUp followers and HijUp.com customers.

Energy in Emotional Es is about Make things more accessible, HjUp.com is a business based online which can be accessed anywhere, from private PC at home to a mobile tablet or smart phone. Make things easier by designed a clean and simple interface when customers conduct online transaction. Make things worthwhile by selling only qualified and high quality products. Make things personalized by using Twitter as an inter alia media, can be used as one-to-one communication tool with consumers. This makes them feel being heard and personalized, because it links directly to their Twitter account which accessed from their palm (smart phone/tablet). This, literally, company is speaking directly to them. Leverage Information and Technology. One of HijUp.com value is empower the consumers. They empower the consumers by get them involved in every stages of transaction they made. Provide security. In this case, HijUp.com provides it consumers by a clear process of transaction. HijUp.com continuously repeats the order and guidance to strengthen the security. The entire explanation beforehand is the answer of this study research question, about the role of Twitter as an inter alia media of HijUp.com. HijUp.com implements all the elements of Emotional Es from Equity, Experience and Energy. This implementation builds a strong emotional relationship which engaged the customers in one, a loyalty.

The Limitations of this Research are the interviews were conduct online, due to the tight schedules of the informants, Researcher cannot schedule a skype interview to fill the lack of face-to-face contact due to the tight schedules of informants and the gadget limitations of each informants and some failed invitation to some targeted customers because of the short period of announcement.

Suggestions for future research are build a strong initial contact not only with the Managing Director but also with the administrator and the targeted followers of a particular account. For future research, another researcher could study the five value of value star or study the Emotional Es at another company or community.

\section{REFERENCES}

\section{Journal Papers:}

[1] Burton and Alena Soboleva. 2011. Interactive or reactive? Marketing with Twitter. Journal of Consumer Marketing Vol 28 No.7, 2011 pp 491-499

[4] Burton and Alena Soboleva. 2011. Interactive or reactive? Marketing with Twitter. Journal of Consumer Marketing Vol 28 No.7, 2011 pp 491-499

[5] Durkin, Mark. Seamas McKenna and Darryl Cummins. 2011. Emotional connections in higher education marketing. International Books: Journal of Educational Management Vol. 26 No. 2, 2012 pp. 153-161

[2] Fill, Chriss. 2009. Marketing Communications: Interactivity, communities and content. Fifth Edition. Prentice Hall.Essex.

[3] Robinette, Scott and Claire Brand with Vicki Lenz. 2001. Emotion Marketing: The Hallmark Way of Winning Customers for Life. Mc Graw Hill. New York.

[6] Grewall, Dhruv and Michael Levy. 2008. Marketing. Mc Graw Hill. New York

[7] Borchers, Timothy A. 2005. Persuasion in the Media Age. Mc Graw Hill. New York.

[8] Sterne, Jim. 2010. Social media metrics : how to measure and optimize your marketing investment. John Wiley and Sons. New Jersey.

[9] Santrock, John W. 2008. Adolescence. Twelfth Edition. Mc Graw Hill. New York.

[10] Creswell, John W. 1994. Research Design: Qualitative and Quantitative approaches. Sage Publications. Thousand Oaks.

[11] Berg, Bruce Lawrence. 2001. Qualitative research methods for the social sciences. Pearson Education Company. USA

[12] Yin, Robert K. 2009. Case Study Research: Design and Methods. Fourth Edition. SAGE Publications. California

[13] Babbie, Earl. 2005. The Basics of Social Research. Third Edition. Thomson and Wadsworth. Canada.

[14] Singleton, Royce A and Bruce C Straits. 2005. Approaches to social research. Oxford University Press, Inc. Madison Avenue. 